\title{
Solitary Renal Metastasis of Follicular Thyroid Carcinoma with Cervical Nodal Deposits
}

\author{
Omar Hamdy ${ }^{1}$, Farida A Shokeir ${ }^{2}$, Gehad A Saleh ${ }^{3}$, Shadi Awni ${ }^{4}$, Marwa MA Zaki ${ }^{5}$
}

\begin{abstract}
Differentiated thyroid cancer includes both papillary thyroid carcinoma and follicular thyroid carcinoma (FTC). It is known to be indolent and is usually limited to the thyroid at the time of presentation. Synchronous case presentation of a thyroid swelling with a regional lymph node and solitary distant renal metastasis is a not so common presentation in FTC. We report the clinical, radiological, and pathological features in addition to management of a case of FTC in a 56-year-old female who presented with a neck swelling and upon diagnostic workup, a solitary renal swelling was discovered, which was diagnosed as metastasis from the original thyroid lesion. Our case shows both regional lymphatic spread and distant hematogenous spread to the kidney. Lymphatic spread has been described in the literature with FTC, but is uncommon. Distant metastasis through hematogenous spread can occur but most often to lungs and bones. Hematogenous metastasis to the kidney is extremely rare.

Aim: To present a very rare presentation of FTC with the kidney being the only site for hematogenous metastasis and conduct a focused literature review on similar cases and illustrate the pathological and molecular bases for diagnosis.

Keywords: Differentiated thyroid cancer, Follicular thyroid carcinoma, Kidney, Metastasis.

World Journal of Endocrine Surgery (2020): 10.5005/jp-journals-10002-1276
\end{abstract}

\section{BACKGROUND}

Differentiated thyroid carcinoma (DTC) originates from follicular cells. It includes both papillary (PTC) and follicular (FTC) carcinomas, which are characterized by being indolent and slowly growing. Follicular thyroid carcinoma is the second most common cancer after PTC in patients over 40 years of age. ${ }^{1}$ Distant metastasis at time of diagnosis has been reported in $11-20 \%$ of patients. ${ }^{2}$ Renal distant metastasis, however, is extremely rare $^{3}$ and has been reported with only a prevalence of $0.47 \%$ of cases. ${ }^{4}$ Lymphatic spread with FTC has been described in $<10 \%$ of cases. ${ }^{5}$ In absence of other metastasis, nephrectomy is reported to be the best therapeutic option rather than radioactive iodine and tyrosine kinase inhibitors, which are commonly used in treatment of recurrence and other metastatic sites such as lungs and bone. ${ }^{6}$

\section{Case Description}

A 56-year-old female, housewife, with medical history of hypertension and insulin-controlled type II diabetes mellitus, and no previous surgical history, presented with lower neck swelling. By clinical examination, she showed hard thyroid swelling associated with enlarged right middle deep cervical lymph nodes. Neck ultrasonography described enlarged both thyroid lobes, which showed multiple bilateral hypoechoic nodules with areas of cystic degeneration and foci of calcifications inside, the largest was in the right lobe measuring $2.2 \times 1.8 \mathrm{~cm}$. Another exophytic mass was described extending from the thyroid capsule to the right sternocleidomastoid muscle. Multiple enlarged deep cervical lymph nodes (LNs) were detected showing distorted shape and lost hilum. Post-contrast neck CT (Fig. 1) showed enlarged both thyroid lobes with multiple hyperdense focal lesions inside, the largest one was seen in the right lobe and isthmus measuring $2.8 \times 3.2 \times 2.4 \mathrm{~cm}$ with foci of calcification inside. It was seen contacting the strap muscles anteriorly with no definite line of separation in-between

\footnotetext{
${ }^{1,4}$ Department of Surgical Oncology, Oncology Center, Mansoura University, Mansoura, Egypt

2,5Department of Pathology, Faculty of Medicine, Mansoura University, Mansoura, Egypt

${ }^{3}$ Department of Radiology, Faculty of Medicine, Mansoura University, Mansoura, Egypt

Corresponding Author: Omar Hamdy, Department of Surgical Oncology, Oncology Center, Mansoura University, Mansoura, Egypt, Phone: +20 1003526752, e-mail: omarhamdy87@gmail.com

How to cite this article: Hamdy O, Shokeir FA, Saleh GA, et al. Solitary Renal Metastasis of Follicular Thyroid Carcinoma with Cervical Nodal Deposits. World J Endoc Surg 2020;12(1):30-33.

Source of support: Nil

Conflict of interest: None
}

with possibility of infiltration. Multiple enlarged upper and lower deep cervical as well as posterior triangle right cervical LNs were detected, the largest showed heterogeneous enhancement measuring $4 \times 2.8 \mathrm{~cm}$. Chest CT showed no detectable abnormality. Post-contrast abdomen CT (Fig. 2) showed left renal well-defined partially exophytic midzonal soft tissue mass completely separable from the surrounding organs measuring $5.5 \times 4.8 \times 4.4 \mathrm{~cm}$. Trucut biopsy was performed from the cervical LN. Microscopic examination revealed follicular cell proliferation arranged in the trabeculae with follicular formation. This led to the diagnosis of infiltration by follicular thyroid neoplasm. Microscopic examination of the fine-needle aspirate (FNA) from the renal mass (Fig. 3) revealed thyroid follicles lined by flat follicular cells and filled with colloid; aggregates of follicular cells and areas of fibrosis were seen as well. This led to the diagnosis of renal infiltration by FTC. The patient was prepared for staged surgery. In the first operation, the patient underwent total thyroidectomy-after difficult dissection of the right lobe from the larynx - and right radical neck dissection 
due to infiltration of the sternomastoid and jugular vein by the nodal mass. Microscopic examination of the thyroidectomy specimen (Fig. 4) revealed neoplastic proliferation of the right thyroid lobe composed of packed microfollicles lined by follicular cells with regular nuclear contours, fine chromatin, and inconspicuous nucleoli and filled with scanty colloid. The neoplastic proliferation showed thyroid extracapsular invasion. Immunohistochemistry (IHC) for cytokeratin 19 (CK19) was performed to exclude the follicular variant of papillary thyroid carcinoma (FVPTC), which was negative. This led to the diagnosis of FTC of the right thyroid lobe extending outside the thyroid capsule. Microscopic examination of the dissected LNs revealed infiltration of 8 out of 16 dissected LNs by the same tumor tissue. The patient was admitted to the intensive care unit for 24 hours for monitoring of vital signs and airway. She was discharged from the hospital after 72 hours with an uneventful postoperative course. Four weeks later, the patient underwent left nephrectomy. By gross examination, the kidney measured $14 \times 6 \times$ $6 \mathrm{~cm}$. It showed a well-circumscribed, round firm mass infiltrating the cortex from outside measuring $5.5 \times 4 \times 2.5 \mathrm{~cm}$. The renal pelvis
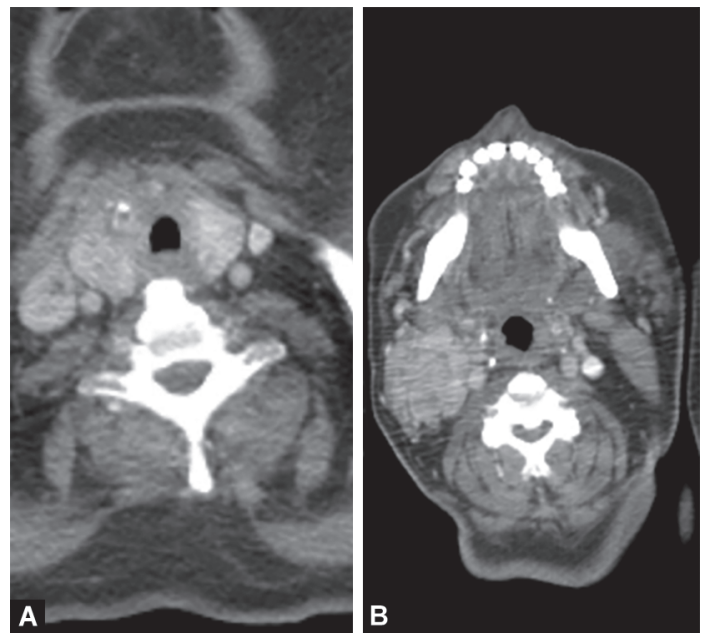

Figs $1 \mathrm{~A}$ and B: Post-contrast CT neck axial images: Asymmetrically enlarged thyroid gland with heterogeneous parenchyma and few nodules, interrupted fat planes between the right lobe and strap muscles. Few distorted right cervical LNs, the largest at level III measures $2.8 \times 4 \mathrm{~cm}$
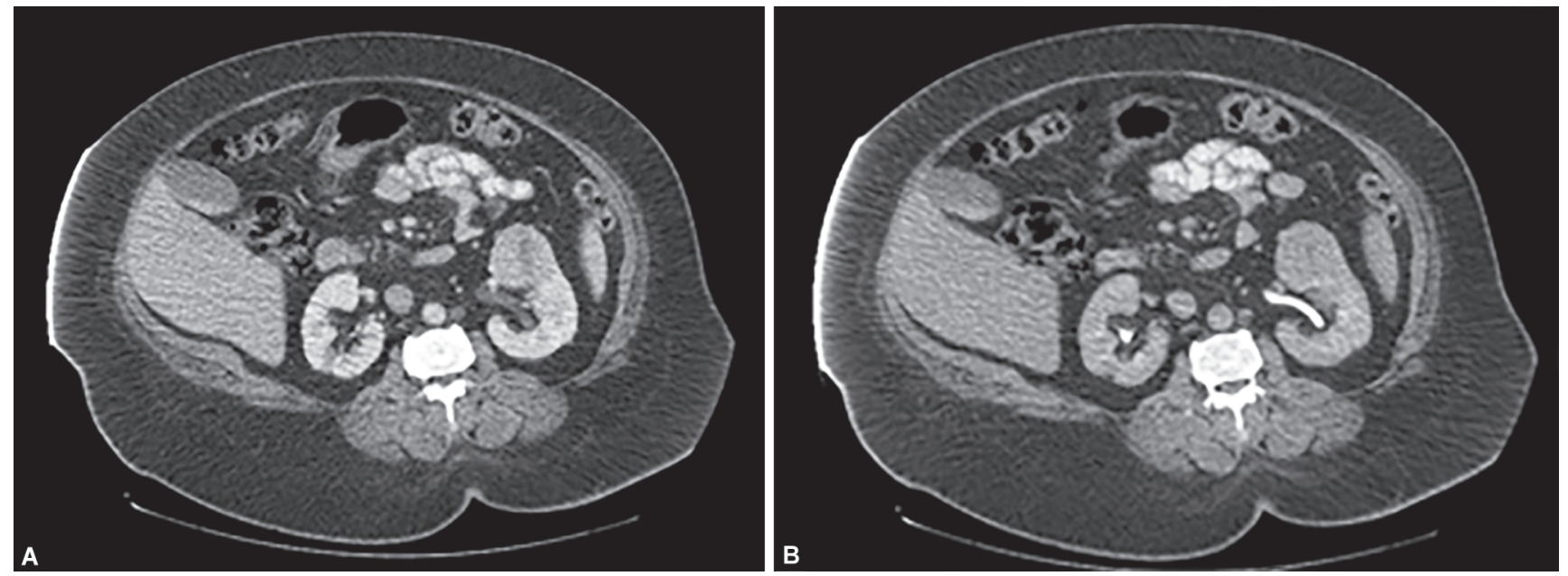

Figs $2 \mathrm{~A}$ and B: Post-contrast CT abdomen axial images portal and delayed phases. Left renal exophytic heterogeneously enhancing soft tissue mass with clear surrounding fat planes, no extension into renal pelvis was grossly free. Sections prepared from the mass (Fig. 5) revealed infiltration of the renal cortical tissue by thyroid follicles lined by follicular cells with rounded nuclei, regular nuclear membranes, and inconspicuous nucleoli lacking characteristic nuclear features of PTC. A fibrous tissue capsule was seen separating the neoplastic proliferation from the surrounding normal kidney. The renal pelvis was infiltrated as well. Immunohistochemistry for thyroid markers revealed positivity for thyroglobulin and the thyroid transcription factor "TTF-1" confirming the thyroid origin and excluding thyroidlike follicular carcinoma of the kidney (TLFCK), which has the same histologic appearance of FTC but lacks expression of thyroid markers. While IHC for CK19 was negative confirming the same histological type of malignancy with that of the thyroid gland. This led to the diagnosis of metastatic FTC in the kidney. She was discharged from the hospital after 60 hours with an uneventful postoperative course. She was later on referred to the clinical oncology department where she received two ablative doses of radioactive iodine. The patient was under regular follow-up for 4 years with no disease recurrence or distant metastasis.

\section{Discussion and Conclusion}

Follicular thyroid carcinoma is the second most common thyroid carcinoma accounting for almost $10 \%$ of all thyroid malignancies. ${ }^{3}$ Follicular thyroid carcinoma is now subclassified according to the latest 2017 version of the World Health Organization (the fourth edition of the WHO classification for thyroid tumors) into three subtypes: minimally invasive, encapsulated angioinvasive, and widely invasive. $^{7}$

Follicular thyroid carcinoma is regarded more aggressive than PTC and often presents with hematogenous distant metastasis to the lung and the bone in $25-30 \%$ of cases rather than PTC, which tends to metastasize to cervical LNs. ${ }^{8-10}$ In FTC, lymphatic spread is much less common but can occur as seen in $<10 \%$ of cases. ${ }^{5}$ The kidney as a rare metastatic site has been reported with only a $0.47 \%$ prevalence. ${ }^{4}$ Two cases have been reported: one by Yamazaki et al. in 2018 and another by Nath et al. in 2015 with previous history of FTC and developed after a period of over two decades of solitary renal metastasis with no reported recurrence or metastasis elsewhere. This case is unique in having lymphatic spread to regional lymph nodes and hematogenous spread at time of initial presentation. 


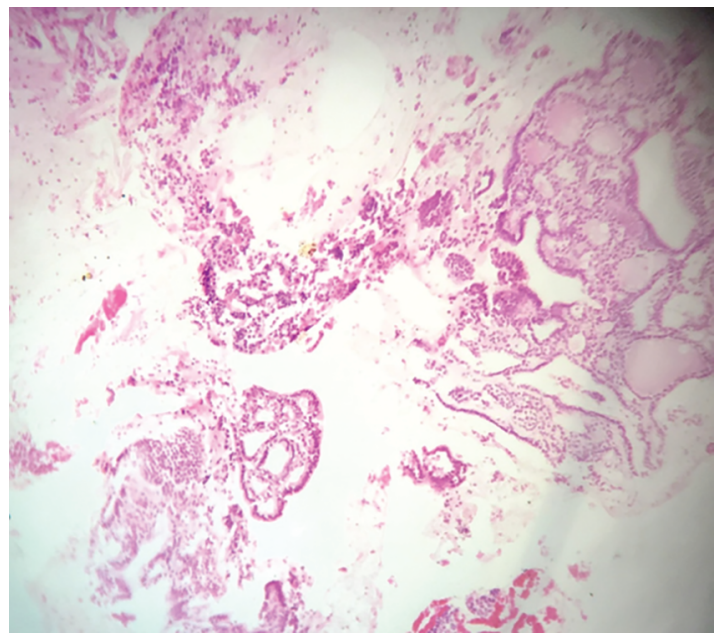

Fig. 3: Fine-needle aspirate from renal mass: thyroid follicles lined by flat follicular cells with evident colloid, seen on a proteinaceous background

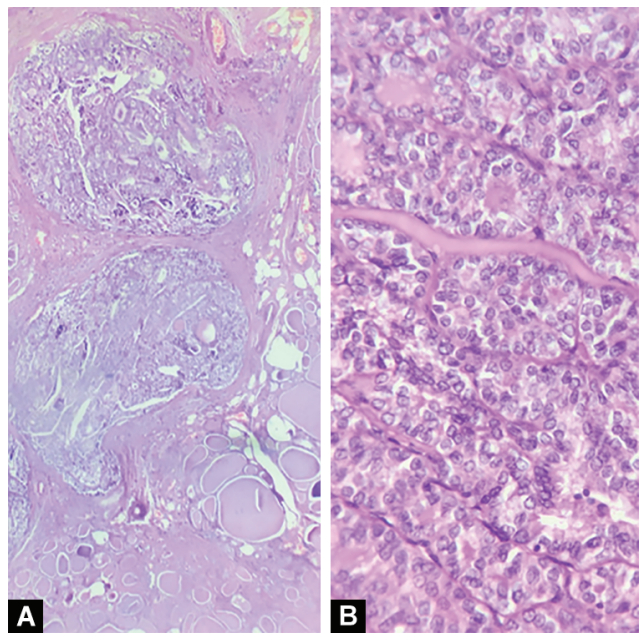

Figs 4A and B: Microscopic examination of the thyroid gland: (A) Three well-circumscribed nodules lined by microfollicles with scanty colloid (Hx\&E, $\times 40)$; (B) Microfollicles lined by monomorphic follicular cells devoid of PTC nuclear features, showing regular nuclear contours, rounded nuclei, and inconspicuous nucleoli $(\mathrm{H} \times \& \mathrm{E}, \times 400)$
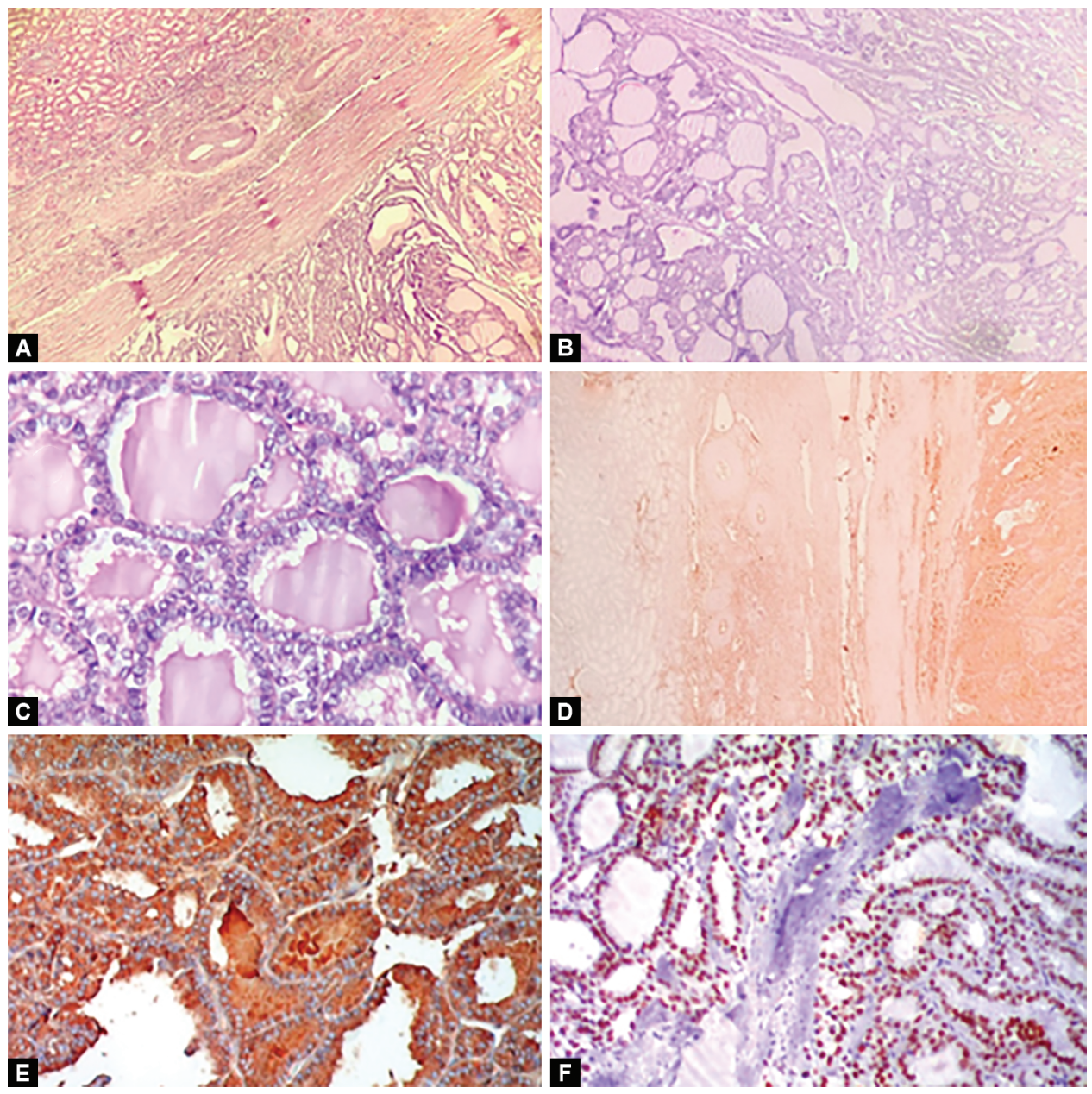

Figs 5A to F: Microscopic examination of the kidney: (A) A section in the normal kidney seen in upper left, lower right, showed infiltration by neoplastic follicular thyroid proliferation surrounded by the fibrous capsule $(\mathrm{Hx \& E}, \times 40)$; (B) Neoplastic thyroid follicles with foci of the trabecular pattern formed of atypical follicular cells lacking nuclear features of PTC (Hx\&E, $\times 100)$; (C) Packed thyroid follicles lined by follicular cells, showing regular nuclear contours, rounded nuclei, and inconspicuous nucleoli (Hx\&E, $\times 400)$; (D) Cytoplasmic staining of thyroglobulin in neoplastic follicular cells infiltrating the kidney on the right side of the image with negative staining in the normal kidney seen on the left side of the image $(X 40)$; $(E)$ Cytoplasmic staining of thyroglobulin in neoplastic follicular cells infiltrating the kidney (intermediate magnification, $\times 100)$; (F) Nuclear staining of TTF-1 in neoplastic proliferation of follicular cells infiltrating the kidney (intermediate magnification, $\times 100$ ) 
Diagnosis of FTC can be confused with the encapsulated follicular variant of PTC (FVPTC). It is similar to FVPTC in having a fibrous capsule and higher hematogenous metastasis potential rather than classic PTC. ${ }^{11}$ It also shares genetic abnormalities with FVPTC, like PAX8/PPAR $\gamma$ rearrangements and RAS mutations. ${ }^{11,12}$

Metastatic thyroid carcinoma to the kidney is usually asymptomatic. Some patients may complain from loin pain or hematuria. In most of the cases, there is a long interval between the initial diagnosis and the diagnosis of renal metastasis. Its presence at the time of initial presentation-like our presented case-is very rare and occurs only in $2 \%$ of cases. ${ }^{6,13}$

Radiologically, metastatic thyroid carcinoma in the kidney does not have pathognomonic radiological findings in the CT or MRI that can differentiate it from renal cell carcinoma. On the other side, histopathological microscopic examination can do, even in the mimickers of metastatic FTC such as thyroid-like follicular carcinoma of the kidney (TLFCK). Immunohistochemical (IHC) stains like thyroglobulin and thyroid transcription factor 1 (TTF-1) show positivity in differentiated thyroid cancer and are negative in cells of the primary renal origin. . $^{614}$

As regard management, surgical resection for the thyroid gland as well as the kidney is the best suggested approach that offers the best survival. It should be followed by radioactive iodine ablative therapy. The patients should be kept on suppressive doses of L-thyroxine and followed up by serum thyroglobulin, neck ultrasound, and iodine scan. ${ }^{15}$

\section{Clinical Significance}

Follicular thyroid carcinoma is a differentiated thyroid cancer, which is known by its hematogenous metastatic potential. However, even patients initially diagnosed as stage IV cancer can enjoy long disease-free and overall survival if adequate surgical management is performed.

\section{ACKnOWLedgments}

To Dr Emad Hamed, MD, Dr Islam Hani, MD, Mohammad Zuhdy, MSc, and Mansoura Elmoatasem, MSc, who are the members of the surgical team who performed the thyroidectomy and the nephrectomy.

\section{References}

1. Mazzaferri EL. Papillary and follicular thyroid cancer: a selective approach to diagnosis and treatment. Annu Rev Med [Internet] 1981;32(1):73-91. DOI: 10.1146/annurev.me.32.020181. 000445.

2. Schlumberger $M$, Tubiana $M$, De Vathaire $F$, et al. Long-term results of treatment of 283 patients with lung and bone metastases from Differentiated thyroid carcinoma. J Clin Endocrinol Metab 1986;63(4):960-967. DOI: 10.1210/jcem-63-4-960.

3. Sampson E, Brierley JD, Le LW, et al. Clinical management and outcome of papillary and follicular (differentiated) thyroid cancer presenting with distant metastasis at diagnosis. Cancer [Internet] 2007;110(7):1451-1456. DOI: 10.1002/cncr.22956.

4. Qiu Z-L, Xue Y-L, Luo Q-Y. Rare renal metastases from differentiated thyroid carcinoma: early clinical detection and treatment based on radioiodine. Arq Bras Endocrinol Metabol 2014;58(3):260-269. DOI: 10.1590/0004-2730000002913.

5. Panda SK, Patro B, Samantaroy MR, et al. Unusual presentation of follicular carcinoma thyroid with special emphasis on their management. Int J Surg Case Rep [Internet] 2014;5(7):408-411. DOI: 10.1016/j.ijscr.2014.03.006. Available from http://www.sciencedirect. com/science/article/pii/S2210261214000522.

6. Yamazaki H, Kishida T, Noguchi G, et al. Nephrectomy for metastatic kidney tumor in patients with differentiated thyroid cancer: a report of two cases. Case Rep Endocrinol 2018;2018:7842792.

7. Lloyd RV, Osamura RY, Klöppel GRJ. WHO Classification of Tumours of Endocrine Organs, 4th ed., Lyon, France: IARC; 2017.

8. D'Avanzo A, Treseler P, Ituarte PHG, et al. Follicular thyroid carcinoma: histology and prognosis. Cancer [Internet] 2004;100(6):1123-1129. DOI: 10.1002/cncr.20081.

9. Iwai $\mathrm{H}$, Ohno $\mathrm{Y}$, Ito $\mathrm{H}$, et al. Renal rupture associated with a poorly differentiated follicular thyroid carcinoma metastasizing to the thigh muscle, lung and kidney. Intern Med 2005;44(8):848-852. DOI: 10.2169/internalmedicine.44.848.

10. Cochetti G, Puxeddu E, Zingaro MD, et al. Laparoscopic partial nephrectomy of thyroid cancer metastasis: case report and review of the literature. Onco Targets Ther [Internet] 2013;6:355-360. DOI: 10.2147/OTT.S37402. Available from https://www.ncbi.nIm.nih.gov/ pubmed/23596352.

11. Sobrinho-Simões $M$, Eloy $C$, Magalhães J, et al. Follicular thyroid carcinoma. Mod Pathol [Internet] 2011;24:S10. DOI: 10.1038/ modpathol.2010.133.

12. Yang GCH, Fried K, Yakoushina TV, et al. Encapsulated follicular variant of papillary thyroid carcinoma: fine-needle aspiration with ultrasound and histologic correlation of 41 cases. Acta Cytol [Internet] 2013;57(1):26-32. Available from https://www.karger.com/ $\mathrm{DOI} / 10.1159 / 000342986$.

13. Falzarano SM, Chute DJ, Magi-Galluzzi C. Metastatic papillary thyroid carcinoma to the kidney: report of two cases mimicking primary renal cell carcinoma and review of the literature. Pathol - J RCPA [Internet] 2013;45(1):89-93. Available from https://journals.Iww.com/ pathologyrcpa/Fulltext/2013/01000/Metastatic_papillary_thyroid_ carcinoma_to_the.17.aspx.

14. Insabato L, Ben-Dor D, Galliani CA, et al. Primary thyroid and thyroidlike follicular carcinoma of the kidney versus solitary metastatic carcinoma of the thyroid: a vexing issue. Virchows Arch [Internet] 2009;454(6):717-718. DOI: 10.1007/s00428-009-0785-z.

15. Lubana SS, Singh N, Tuli SS, et al. Follicular variant of papillary thyroid cancer with bilateral renal Metastases discovered incidentally during work-up of primary Endometrial cancer: a rare occurrence. Am J Case Rep [Internet] 2015;16:459-468. DOI: 10.12659/AJCR.894935. Available from https://www.ncbi.nlm.nih.gov/pubmed/26181765. 